\title{
Hábitos alimentarios del tiburón zorro Alopias superciliosus (Lowe, 1839), en el Pacífico ecuatoriano
}

\author{
Food habits of thresher shark Alopias superciliosus (Lowe, 1839) in Ecuadorian Pacific
}

\section{Carlos Polo-Silva ${ }^{1}$, Álvaro Baigorrí-Santacruz ${ }^{1}$, Felipe Galván-Magaña ${ }^{2}$, Marcela Grijalba-Bendeck ${ }^{1}$ y Adolfo Sanjuan-Muñoz ${ }^{1}$}

\author{
${ }^{1}$ Facultad de Ciencias Naturales, programa de Biología Marina, Universidad de Bogotá Jorge Tadeo Lozano \\ Carrera 2 \# 11-68, Edificio Mundo Marino, El Rodadero. Santa Marta, Colombia \\ ${ }^{2}$ Centro Interdisciplinario de Ciencias Marinas. Av. I. P. N. s/n Colonia Playa Palo de Santa Rita, apartado postal 592.23000 \\ La Paz, Baja California Sur, México \\ marcela.grijalba@utadeo.edu.co
}

Resumen.- Se analizó el espectro trófico de 122 tiburones zorro Alopias superciliosus capturados artesanalmente en playa Tarqui, Manta (Ecuador), entre julio y diciembre de 2003. Los individuos fueron categorizados por sexo y estados de madurez (maduro e inmaduro) y sus contenidos estomacales se fijaron y separaron por componentes. Se aplicaron índices tróficos cuantitativos (numérico, gravimétrico y frecuencia de ocurrencia) que se analizaron en la escala temporal. Se encontraron 27 componentes alimentarios, siendo las principales presas Larimus argenteus $(\mathrm{IIR}=1497)$, Merluccius gayi $(\mathrm{IIR}=333)$, Dosidicus gigas $(\mathrm{IIR}=281)$ y Benthosema panamense (IIR=239). El índice de Levin permitió definir a A. superciliosus como un depredador especialista, presentando tendencia a alimentarse en zonas oceánicas.

Palabras clave: Alopias superciliosus, Alopiidae, contenido estomacal, ecología trófica, Ecuador

\begin{abstract}
The feeding habits of the common thresher shark (Alopias superciliosus) from the Pacific coast of Ecuador were investigated by using frequency of ocurrence, numeric and gravimetric methods. A total of 122 individuals were collected from July to December 2003 and grouped by sex and maturity state (mature vs. inmature). Stomach contents were examined and classified by components to infer trophic relations. A total of 27 prey taxa were observed as part of the diet of Alopias superciliosus. Overall, Larimus argenteus (RII=1497) was the most important in the diet, followed by Merluccius gayi (RII=333), Dosidicus gigas (RII=281) and Benthosema panamense (RII=239). The Levin's index allowed to define $A$. superciliosus as an specialist predator that feeds in oceanic waters.
\end{abstract}

Key words: Alopias superciliosus, Alopiidae, stomach content, trophic ecology, Ecuador

recursos pesqueros, creando estrategias que contribuyan a su correcta administración (Galván et al. 1989).

El tiburón zorro Alopias superciliosus vive en aguas costeras sobre la plataforma continental y en zonas someras cercanas a la costa; es nadador activo, resistente, epipelágico y al mismo tiempo epibentónico, que frecuenta la columna de agua desde la superficie hasta por lo menos $500 \mathrm{~m}$ de profundidad (Compagno 2001). Se cree que posee hábitos alimentarios especializados ya que ataca cardúmenes de peces y calamares nadando alrededor de ellos en forma circular y golpeándolos con su aleta caudal (Compagno 1984). Debido a su amplia distribución, elevada abundancia y al alto valor comercial, se explota en el océano Índico 
noroccidental (Japón y Rusia) y en el Pacífico central (Cuba). Generalmente la carne se comercializa para el consumo humano, el hígado para la extracción de vitaminas, la piel se emplea en la fabricación de artículos de cuero y las aletas para la preparación de alimentos (Compagno 2001).

Ecuador se caracteriza por ser uno de los países que más aprovecha sus recursos marinos, el puerto de Manta ubicado en el norte de la provincia de Manabí, es uno de los centro de acopio pesquero artesanal e industrial más importante del país, considerado así por los altos volúmenes de pesca que registra (alrededor de 8500 t semestrales), conformada por diversas especies comerciales, entre ellas muchas de tiburones. En Manta se encuentra el mayor índice de desembarque de tiburones zorro A. pelagicus y A. superciliosus, representando el $37 \%$ del total de capturas en aguas ecuatorianas (Alcívar \& López 2001).

Actualmente en Ecuador no existen estudios sobre la biología básica de A. superciliosus, sólo se conocen informes de la pesquería artesanal e industrial (Instituto Nacional de Pesca de Ecuador 1998, Martínez 1998, López et al. 1999). A nivel mundial A. superciliosus ha sido objeto de diversos trabajos encaminados al conocimiento de sus aspectos reproductivos y características morfológicas (Moreno \& Morón 1992, Chen 1997), así como en determinaciones de edad y crecimiento (Liu et al. 1999). El objetivo de esta investigación es determinar el espectro trófico del tiburón zorro Alopias superciliosus, mediante un análisis cuantitativo de los componentes alimentarios, considerando aspectos como el sexo y estados de madurez.

\section{Material y métodos}

Las muestras fueron tomadas en playa Tarqui, Manta (Ecuador), localizada a $0^{\circ} 56^{`} \mathrm{~S}, 80^{\circ} 43^{`} \mathrm{~W}$ (Fig. 1). A partir de los muestreos realizados entre julio $\mathrm{y}$ diciembre de 2003, semanalmente se recolectaron de 5 a 10 tiburones extraídos artesanalmente. A cada tiburón se le midió la longitud total y precaudal, se estableció el sexo y estado de madurez (maduro e inmaduro) según los criterios propuestos por los autores y teniendo en cuenta en los machos la longitud de los clásper, grado de rotación, condición del rifiodón y presencia de semen. Los machos maduros presentaron clásper totalmente calcificados con una rotación mayor a $360^{\circ}$, el rifiodón abierto y presencia de semen. En el caso de las hembras maduras se evidenciaron marcas de cópula en aletas y dorso, abertura vaginal mayor o igual a $3 \mathrm{~cm}$ de diámetro y el peso del ovario por encima de $100 \mathrm{~g}$, las hembras grávidas además de compartir estas características se diferenciaron por la presencia de huevos y embriones en los úteros.

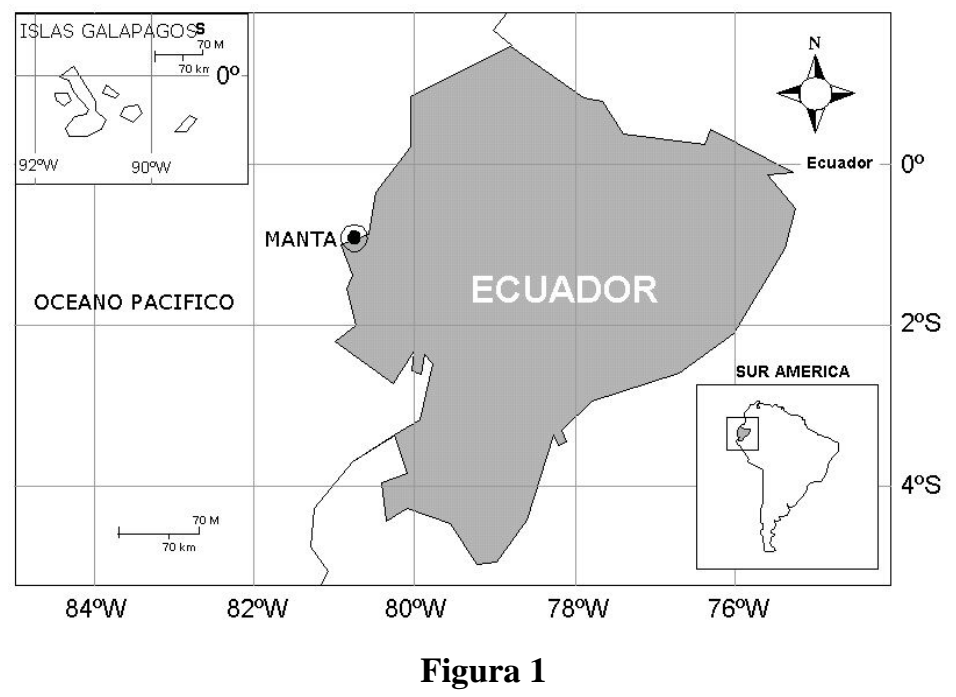

Ubicación del Puerto de Manta, costa ecuatoriana

Geographical location of Manta’s harbor at Ecuadorian coast 
Para la obtención del contenido estomacal se extrajo el estómago realizando un corte ventral al tiburón desde la cloaca hasta el centro de las aletas pectorales. El estómago se cortó desde la altura del esófago hasta la válvula espiral, luego se separó y se calculó la proporción de llenado de acuerdo a la escala propuesta por Galván et al. (1989). Posteriormente el contenido estomacal fue refrigerado hasta su análisis en laboratorio. Se separaron y fijaron (formol 10\%) las presas por grupos mayores, en el caso de los peces en avanzado estado de digestión la determinación taxonómica se realizó con base en las características del esqueleto axial y los otolitos. Para el conteo de vértebras se utilizaron los trabajos de Clothier (1950) y Clothier \& Baxter (1969), para otolitos García (2001); los peces en estado de digestión mínima se identificaron siguiendo a Chirichigno (1998) y Fischer et al. (1995); los crustáceos con las claves de Garth \& Stephenson (1966) y los cefalópodos usando Clarke (1962, 1986) y Wolff (1982, 1984). Las medidas mandibulares de Clarke (1986) y Wolff (1984) se emplearon para identificar los cefalópodos a partir de sus picos.

En el análisis cuantitativo de los contenidos gástricos se utilizaron los métodos numérico $(\mathrm{N})$, gravimétrico (expresado en gramos) y frecuencia de ocurrencia (FO) propuesto por Hyslop (1980). El índice de importancia relativa (IIR) propuesto por Pinkas et al. (1971) se empleó para analizar las variaciones mensuales de la dieta por sexo y estado de madurez. La diversidad de presas se calculó con el índice de Shannon-Wiener ( $\mathrm{H}^{\prime}$ ) y la amplitud de la dieta mediante el índice estandarizado de Levin (Krebs, 1989) expresado por Labropoulou \& Eleftheriou (1997) como:

$$
B i=1 / n-1\left\{\left(1 / \sum J P i j^{2}\right)-1\right\}
$$

Donde $B i$ = Índice de Levin para el predador $j, P i j=$ proporción de la dieta del depredador $i$ sobre la presa $j$, $n=$ número de categorías de presas. Los valores de este índice fluctúan de 0 a 1, por debajo de 0,6 indican una dieta dominada por pocas presas, por lo tanto se trata de un depredador especialista y valores mayores a 0,6 revelan dietas de depredadores generalistas (Krebs 1989, Labropoulou \& Eleftheriou 1997).

Para evaluar el traslapamiento del espectro trófico entre sexos y estados de madurez, se aplicó el índice de Morisita-Horn (Smith \& Zaret 1982):

$$
C \lambda=2 \sum_{i=1}^{n}(P x i * P y i) /\left(\sum_{i=1}^{n} P x i^{2}+\sum_{i=1}^{s} P y i^{2}\right)
$$

Donde $C \lambda=$ índice de Morisita-Horn entre sexo o estado de madurez $x$ y entre sexo o estado de madurez y, $P x i=$ proporción de presa $i$ del total de presas consumidas por el sexo o estado de madurez $x, P y i=$ Proporción de presa $i$ del total de presas consumidas por el sexo o estado de madurez $y, n=$ Número total de presas. Este índice varia entre 0 y 1 y se considera como un traslape biológicamente significativo cuando el valor excede 0,6 (Langton 1982).

\section{Resultados}

\section{Composición de la dieta}

Se analizaron 122 estómagos de A. superciliosus, de los cuales el 87,7\% (107 estómagos) se encontró con alimento o restos de este parcialmente digerido y el 12,2\% (15 estómagos) se encontraron vacíos. La distribución mensual respecto al número de estómagos llenos varió, con los valores más altos en los meses de julio (33 llenos, 13 vacíos), agosto (20 llenos, 5 vacíos) y octubre (21 llenos, 1 vacío), posiblemente debido a la abundancia de la especie, los menores registros se obtuvieron en noviembre (4 llenos) y diciembre (8 llenos, 1 vacío) lo cual puede corresponder a que durante estos meses la abundancia de la especie disminuyó en el área (Fig. 2). La mayoría de los estómagos se encontraron entre un 25\% y $75 \%$ de

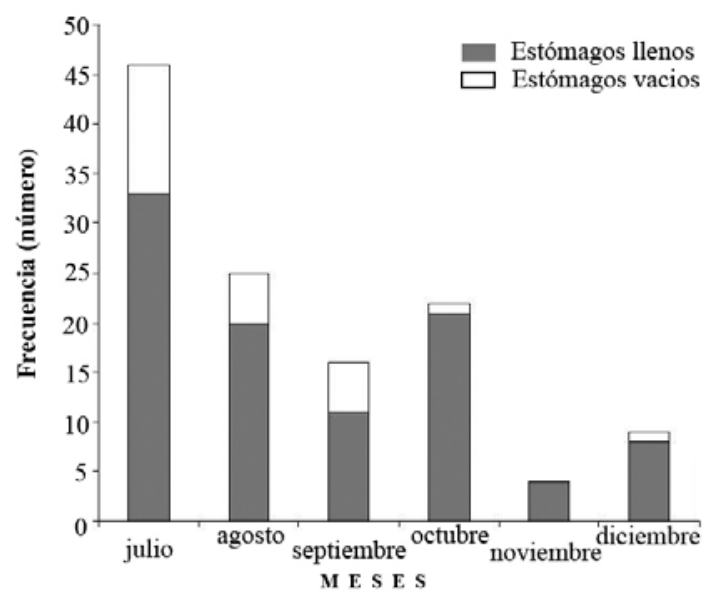

Figura 2

Variación mensual del número de estómagos analizados de Alopias superciliosus en Manta, Ecuador

Monthly variation in the number of analyzed stomachs of Alopias superciliosus at Manta, Ecuador 
Tabla 1

Espectro trófico del tiburón zorro Alopias superciliosus, en Manta Ecuador; expresado en valores absolutos y porcentuales de los métodos numérico (N), gravimétrico (P), frecuencia de ocurrencia (FO), índice de importancia relativa (IIR) C: Presa Circunstancial, S: Secundaria y P: Principal

Trophic spectrum of pelagic thresher Alopias superciliosus at Manta, Ecuador, expressed in absolute and percent values of numerical methods $(\mathrm{N})$, gravimetrical $(\mathrm{P})$, occurrence frequency $(\mathrm{OF})$, and relative importance index (RII)

C: Circumstantial, S: Secondary and P: Main prey

\begin{tabular}{|c|c|c|c|c|c|c|c|c|}
\hline Especie presa & Número & $\% \mathrm{~N}$ & Peso (g) & \% Peso & FO & $\%$ FO & IIR & Categoría \\
\hline Sardinops sagax & 74 & 9,2 & $1.114,7$ & 2,6 & 7 & 7,3 & 76 & $\mathrm{~S}$ \\
\hline Benthosema panamense & 305 & 38,0 & 295,5 & 0,3 & 6 & 6,3 & 239 & $\mathrm{P}$ \\
\hline Morfotipo 5 (Lutjanidae) & 1 & 0,1 & 32,0 & 0,0 & 1 & 1,0 & 0 & $\mathrm{C}$ \\
\hline Auxis thazard & 13 & 1,6 & $1.387,0$ & 3,2 & 13 & 13,5 & 65 & $\mathrm{~S}$ \\
\hline Hemanthias signifier & 1 & 0,1 & 508,1 & 0,6 & 1 & 1,0 & 1 & $\mathrm{C}$ \\
\hline Coryphaena hippurus & 2 & 0,2 & 45,0 & 0,1 & 1 & 1,0 & 0 & $\mathrm{C}$ \\
\hline Larimus argenteus & 252 & 31,3 & $6.588,6$ & 15,1 & 37 & 38,5 & 1497 & $\mathrm{P}$ \\
\hline Remora remora & 1 & 0,1 & 25,6 & 0,0 & 1 & 1,0 & 0 & $\mathrm{C}$ \\
\hline Ophichthus sp. & 1 & 0,1 & 110,0 & 0 , & 1 & 1,0 & 0 & $\mathrm{C}$ \\
\hline Morfotipo 2 & 1 & 0,1 & 24,0 & 0,0 & 1 & 1,0 & 0 & $\mathrm{C}$ \\
\hline Morfotipo 3 & 3 & 0,4 & 53,1 & 0,1 & 2 & 2,1 & 1 & $\mathrm{C}$ \\
\hline Fistularia corneta & 1 & 0,1 & 130,0 & 0,1 & 1 & 1,0 & 0 & $\mathrm{C}$ \\
\hline Fistularia sp. & 1 & 0,1 & 30,0 & 0,0 & 1 & 1,0 & 0 & $\mathrm{C}$ \\
\hline Lagocephalus lagocephalus & 2 & 0,2 & 373,5 & 0,4 & 2 & 2,1 & 1 & $\mathrm{C}$ \\
\hline Merluccius gayi & 74 & 9,2 & $2.315,2$ & 5,3 & 27 & 28,1 & 333 & $\mathrm{P}$ \\
\hline Morfotipo 6 (Exocoetidae) & 1 & 0,1 & 8,7 & 0,0 & 1 & 1,0 & 0 & $\mathrm{C}$ \\
\hline Morfotipo 7 (Trachipteridae) & 1 & 0,1 & 25,0 & 0,0 & 1 & 1,0 & 0 & $\mathrm{C}$ \\
\hline Ablennes hians & 5 & 0,6 & 899,0 & 1,0 & 1 & 1,0 & 2 & $\mathrm{C}$ \\
\hline Morfotipo 8 (Scorpaenidae) & 1 & 0,1 & 5,5 & 0,0 & 1 & 1,0 & 0 & $\mathrm{C}$ \\
\hline Subtotal & 740 & 91,8 & 13.971,1 & 16,0 & & & & \\
\hline Dosidicus gigas & 19 & 2,4 & $19.266,4$ & 44,0 & 11 & 11,5 & 281 & $\mathrm{P}$ \\
\hline Sthenoteuthis oualaniensis & 4 & 0,5 & $10.242,6$ & 23,0 & 2 & 2,1 & 26 & $\mathrm{~S}$ \\
\hline Octopodoteuthis sicula & 2 & 0,2 & 0,2 & 0,0 & 1 & 1,0 & 0 & $\mathrm{C}$ \\
\hline Mastigoteuthis dentata & 3 & 0,4 & 0,03 & 0,0 & 1 & 1,0 & 0 & $\mathrm{C}$ \\
\hline Histioteuthis sp. & 1 & 0,1 & 0,2 & 0,0 & 1 & 1,0 & 0 & $\mathrm{C}$ \\
\hline Abraliopsis affinis & 20 & 2,5 & 39,4 & 0,0 & 4 & 4,2 & 11 & $\mathrm{C}$ \\
\hline Ancistrocheirus lesueurii & 16 & 2,0 & 2,3 & 14,6 & 14 & 14,6 & 29 & $\mathrm{~S}$ \\
\hline Subtotal & 65 & 8,1 & 29.551,2 & 34 & & & & \\
\hline Solenocera agassizi & 1 & 0,1 & 23,8 & 0 & 1 & 1,0 & 0 & $\mathrm{C}$ \\
\hline Subtotal & 1 & 0,1 & 23,8 & $\mathbf{0}$ & & & & \\
\hline Total & 806 & & $43.546,1$ & & & & & \\
\hline
\end{tabular}

llenado, el 49\% de las presas se categorizó en estado de digestión 4, el $36 \%$ en 3 y el $15 \%$ en estado 2, lográndose identificar un total de 27 componentes alimentarios agrupados en tres categorías principales crustáceos, cefalópodos y teleósteos (Tabla 1).
Se cuantificaron 806 ejemplares dentro de los contenidos estomacales que representaron una biomasa de 43546,1 g, los cefalópodos aportaron 67,8\% (29.551,2 g), los peces 32,0\% (13.971,1 g) y 0,05\% los crustáceos (23,8 g). El método numérico determinó que 
la presa más representativa fue el pez linterna Benthosema panamense (38,0\%; $\mathrm{n}=305)$, seguido por el scianido Larimus argenteus (31,3\%; $\mathrm{n}=252)$, Sardinops sagax $(9,2 \% ; n=74)$ y Merluccius gayi $(9,2 \% ; n=74)$ (Tabla 1). Gravimétricamente los componentes más importantes fueron Dosidicus gigas (44,0\%; 19.266,4 g) seguido de Sthenoteuthis oualaniensis (23,0\%; $10.242,6$ g), L. argenteus (15,1\%; 6.588,6 g), M. gayi (5,3\%; 2.315,2 g), Auxis thazard (3,2\%; 1.387,7 g) y $S$. $\operatorname{sagax}(2,6 \% ; 1.114,7 \mathrm{~g})$.

Las presas más frecuentes fueron L. argenteus en 37 estómagos (38,5\%), M. gayi en 27 (28,1\%), Ancistrocheirus lesueurii en 14 (14,6\%), A. thazard en 13 (13,5\%), D. gigas en 11 (11,5\%) y S. sagax en 7 (7,3\%). El IIR indicó a L. argenteus (IIR=1.497), $M$. gayi $(\mathrm{IIR}=333)$, D. gigas $(\mathrm{IIR}=281)$ y $B$. panamense $(\mathrm{IIR}=239)$, como los cuatro componentes principales en la dieta de $A$. superciliosus (Tabla 1 ).

\section{Espectro trófico mensual}

Se encontraron fluctuaciones en la dieta de $A$. superciliosus; de julio a septiembre se halló una dominancia de tres presas principales: L. argenteus, $M$. gayi y D. gigas y presas secundarias como $B$. panamense, A. thazard y $S$. sagax. Durante los meses de octubre a diciembre se observó un mayor consumo de $B$. panamense y los cefalópodos $D$. gigas y $S$. oualaniensis. Así mismo aumentó el consumo por presas bentónicas como M. gayi (Fig. 3).

\section{Espectro trófico por sexo y estado de madurez}

De los estómagos recolectados (122), 78 correspondieron a individuos maduros (39 hembras y 39 machos), 27 a inmaduros (7 hembras y 20 machos) y 17 a hembras grávidas. La longitud total de las hembras osciló entre 1,9 y 3,8 m y su alimentación estuvo conformada por 22 componentes alimentarios en 57 estómagos analizados. Las presas principales fueron: L. argenteus, D. gigas, $M$. gayi y $B$. panamense, y la única presa secundaria fue $S$. sagax (Fig. 4). Los machos con longitudes totales (LT) entre 1,35 y 3,27 m presentaron una dieta conformada por 14 componentes en 50 estómagos, las presas principales fueron $L$. argenteus y $M$. gayi, y como secundarias $D$. gigas, A. thazard, S. sagax, B. panamense, A. lesueurii, S. oualaniensis y A. affinis (Fig. 4).

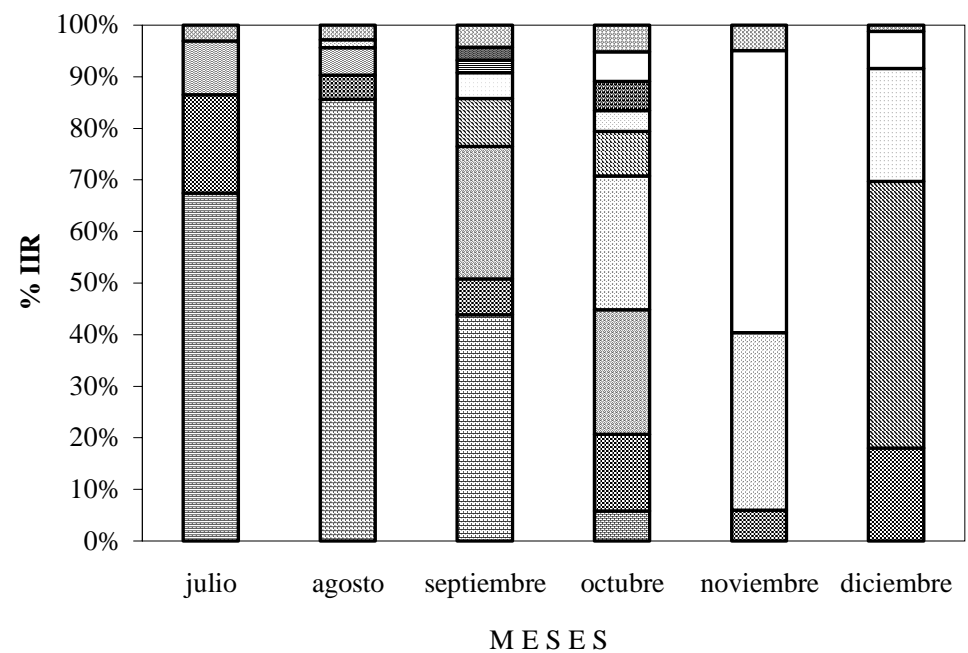

\begin{tabular}{|c|c|c|c|}
\hline 四 L. argenteus & ㄱ. gayi & 图 D. gigas & ㄱ.panamense \\
\hline$g a x$ & $\square$ A. thazard & 目Ophicthus sp 1 & 시⿱ L. lagocephahis \\
\hline A. hians & $\square$ S. oualaniensis & 田ros & \\
\hline
\end{tabular}

Figura 3

Índice de importancia relativa (IIR) de las presas principales del tiburón zorro Alopias superciliosus en Manta Ecuador 


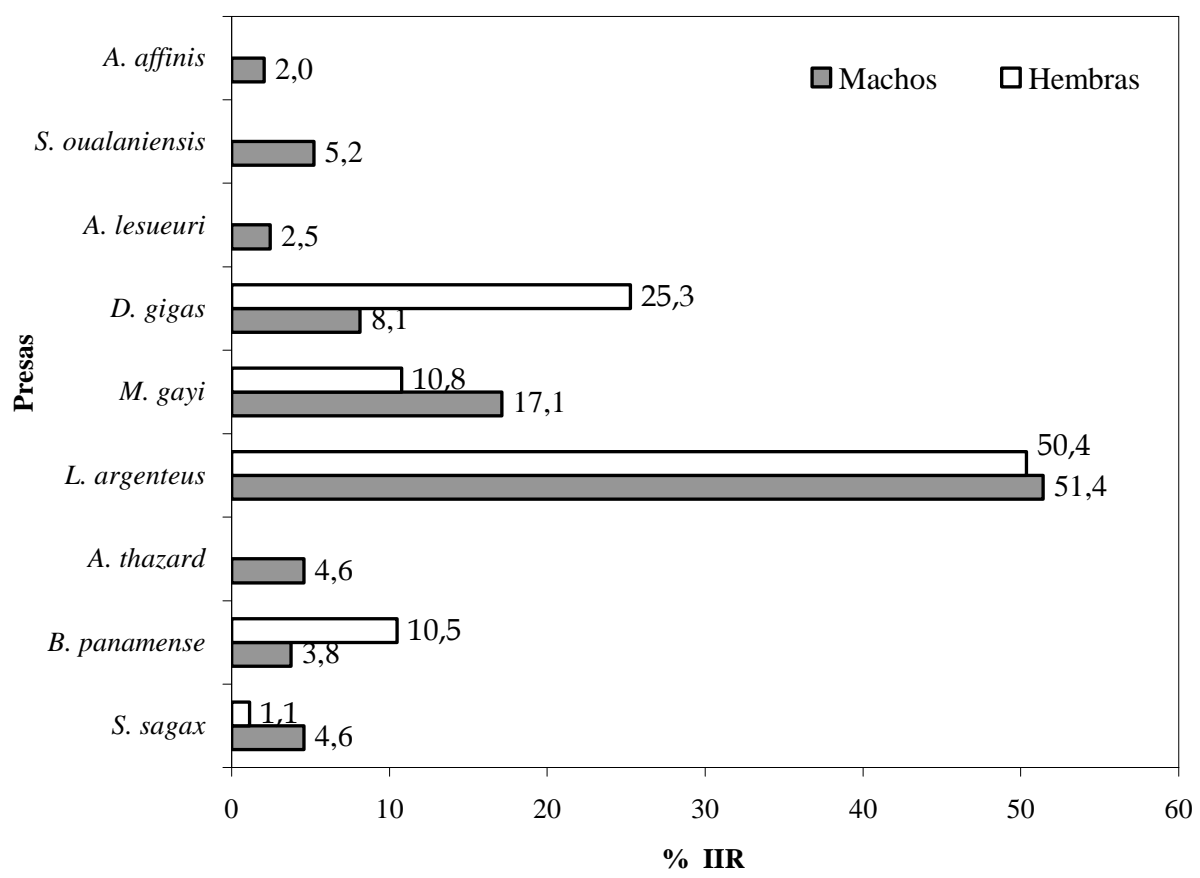

Figura 4

Índice de importancia relativa (IIR) de las presas principales de machos y hembras del tiburón zorro Alopias superciliosus en Manta Ecuador

Relative importance index (RII) of main preys of males and females of bigeye thresher shark Alopias superciliosus at Manta, Ecuador

$\mathrm{Al}$ comparar la dieta entre ejemplares maduros e inmaduros se determinó que las hembras maduras con una talla media de madurez de 2,8 $\mathrm{m}$ (LT), presentaron una dieta conformada por 20 componentes, donde los más importantes según el IIR fueron $L$. argenteus, $B$. panamense, M. gayi y D. gigas (Fig. 5). Las hembras inmaduras con tallas entre 2,1 y 2,6 m (LT) mostraron un espectro trófico constituido por $L$. argenteus, $S$. oualaniensis y M. gayi (Fig. 5). Los machos maduros $(\mathrm{n}=39)$, con una talla media de madurez de 2,6 m (LT), presentaron 13 componentes, siendo las presas principales según el IIR $L$. argenteus, $M$. gayi y $S$. sagax (Fig. 5). En los machos inmaduros con tamaños entre 1,3 y 2,9 m (LT) se evidenciaron seis presas, siendo las principales D. gigas y L. argenteus (Fig. 5). La alimentación de las hembras grávidas estuvo conformada por 16 componentes en 17 estómagos con alimento, las presas principales con base en el IIR fueron $L$. argenteus, B. panamense, $M$. gayi y $A$. thazard (Fig. 6).

\section{Diversidad de presas, amplitud del nicho trófico y traslapamiento de la dieta}

Para A. superciliosus la diversidad promedio de presas fue baja con respecto a la diversidad máxima tanto a nivel general $(0,34 \pm 0,04)$, por sexos y estados de madurez, como para los machos maduros $(0,42 \pm 0,06)$ e inmaduros $(0,15 \pm 0,08)$ y para las hembras maduras $(0,36 \pm 0,07)$ e inmaduras $(0,11 \pm 0,10)$ (Tabla 2). En relación al nicho trófico se encontraron valores bajos tanto para machos como para hembras $(0,21$ y 0,16 respectivamente); de igual forma los valores fueron pequeños de acuerdo al estado de madurez, tanto para las hembras maduras e inmaduras $(0,13$ y 0,24 respectivamente) y para machos maduros e inmaduros $(0,06$ y 0,13$)$. Los valores de traslapamiento trófico para A. superciliosus entre los estados de madurez (maduros e inmaduros) de los machos y las hembras variaron. Se encontró traslapamiento alto entre machos $\mathrm{y}$ hembras $(0,74)$, al igual que entre machos maduros e inmaduros $(0,80)$; sin embargo, este fue bajo entre hembras maduras e inmaduras $(0,33)$. 

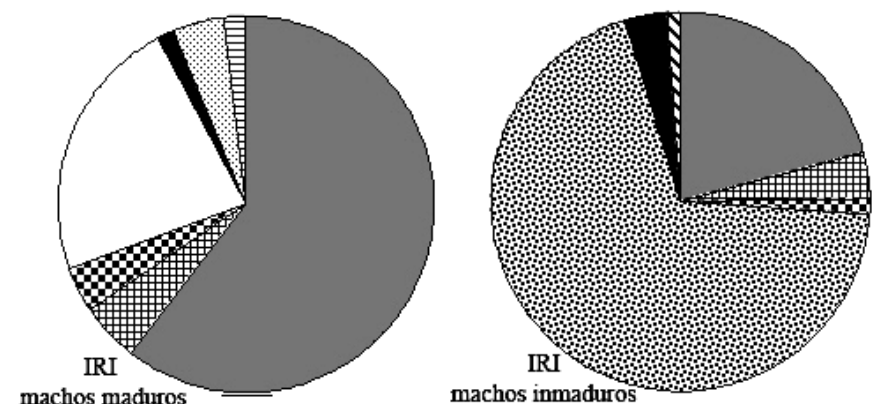

$\square$ L. argenteus

A. hians

目A. affinis

田 A. thazard

몽 gigas

machos maduros

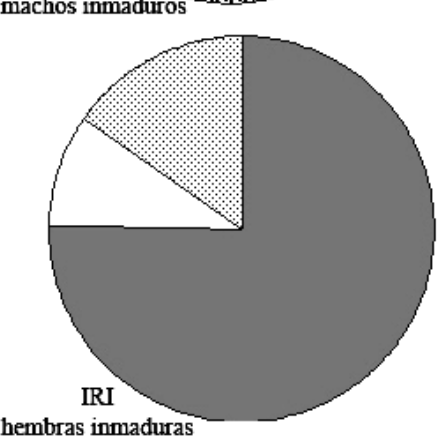

$\mathbf{S}$. evermanni

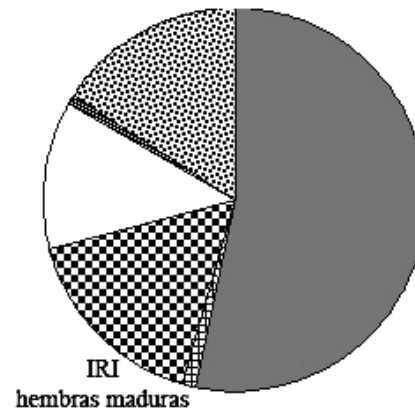

Q B. panamense

A. lesueuri

$\square$ M. gayi

圆 $S$. oualaniensis

\section{Figura 5}

Índice de importancia relativa (IIR) de las presas principales por estados de madurez para hembras y machos del tiburón zorro Alopias superciliosus en Manta, Ecuador

Relative importance index (RII) of main preys for maturity stages for females and males of thresher shark Alopias superciliosus at Manta, Ecuador

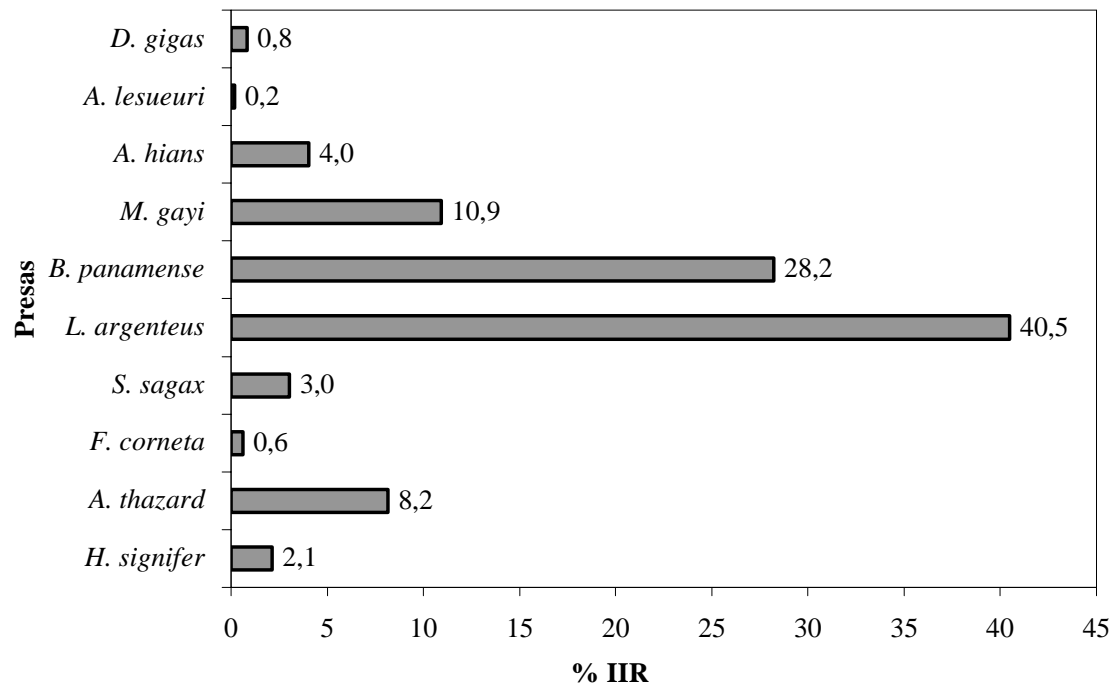

Figura 6

Valor porcentual de las principales presas de hembras grávidas de tiburón zorro Alopias superciliosus en Manta, Ecuador Percent value of main preys of gravid females of pelagic thresher Alopias pelagicus at Manta, Ecuador 


\section{Tabla 2}

Valores de diversidad cuantificados en la dieta del tiburón zorro Alopias superciliosus en Manta, Ecuador

Average diversity values quantified in pelagic thresher Alopias superciliosus at Manta, Ecuador

\begin{tabular}{lcc}
\hline \multicolumn{1}{c}{ Tiburones } & $\begin{array}{c}\text { Shannon- } \\
\text { Wiener }\end{array}$ & $\begin{array}{c}\text { Diversidad } \\
\text { máxima }\end{array}$ \\
\hline General & $0,34 \pm 0,04$ & 3,4 \\
Hembras & $0,35 \pm 0,06$ & 3,2 \\
Machos & $0,34 \pm 0,06$ & 2,9 \\
Hembras maduras & $0,36 \pm 0,07$ & 3,0 \\
Hembras inmaduras & $0,11 \pm 0,1$ & 1,4 \\
Machos maduros & $0,42 \pm 0,06$ & 2,5 \\
Machos inmaduros & $0,15 \pm 0,08$ & 2,4 \\
\hline
\end{tabular}

\section{Discusión}

Los estudios sobre interacciones tróficas son muy importantes desde el punto de vista biológico ya que conducen al entendimiento de las relaciones dentro y entre las especies presa y depredadoras, esto es especialmente valioso cuando el objeto de estudio son especies de interés comercial (Wooton 1990). El análisis de los contenidos estomacales de $A$. superciliosus evidenció que la dieta esta basada en cuatro presas principales: el sciánido $L$. argenteus, la merluza $M$. gayi, el calamar gigante $D$. gigas y el pez linterna B. panamense. La importancia de L. argenteus como presa dentro del espectro trófico de este depredador puede deberse a que esta especie se caracteriza por encontrarse asociada a fondos por encima de los $100 \mathrm{~m}$, habitando en sustratos blandos de la plataforma continental en regiones tropicales y subtropicales (Cervigón \& Álcala 1999), así como en la zona pelágica, con tendencia a permanecer en aguas y lagunas costeras en donde se alimenta principalmente de crustáceos (Chirichigno 1998). Al ser una especie que comparte el mismo hábitat de A. superciliosus tanto en la zona oceánica como en aguas costeras esta en continuo contacto con el depredador, convirtiéndose en una de las presas predilectas. El método de caza de esta especie por parte del tiburón consiste según Compagno (2001) en aturdir sus presas mediante un coletazo, ahorrando así energía al disminuir el esfuerzo de captura.
M. gayi, que se constituyó en la segunda presa en la dieta del tiburón zorro, está presente desde aguas costeras (50 $\mathrm{m}$ de profundidad) hasta el talud continental con una profundidad mayor de $500 \mathrm{~m}$, manteniendo una estrecha relación con el fondo costero y el talud continental. Esta especie se alimenta de especies bentónicas, pero se caracteriza por realizar migraciones verticales alimentándose también de especies pelágicas durante la noche (Castillo et al. 1996). La gran facilidad que tiene esta presa de encontrarse en diversos hábitats y poder sumergirse a grandes profundidades, le permite entrar en el "home range" de A. superciliosus convirtiéndose de esta forma en una de sus presas. Espinoza (2001) resalta que $M$. gayi se encuentra en el borde y fuera de la plataforma continental, con una distribución de continuidad horizontal desde los $200 \mathrm{~m}$, de igual forma existe evidencia que este comportamiento migratorio lo realiza a mayores profundidades y a zonas de difícil acceso, por ello $M$. gayi es una presa común en el hábitat de este depredador, que tiene estrecha relación con el fondo marino, por lo cual se deduce que $A$. superciliosus es una especie que captura sus presas a diferentes profundidades cercanas al fondo.

Los cefalópodos y los peces linterna también fueron importantes dentro de la dieta de A. superciliosus como presas principales a pesar de no tener un valor muy alto, lo cual puede deberse a que este tiburón presenta una mayor tendencia a alimentarse fuera de la plataforma continental, mientras que estas presas se caracterizan por realizar migraciones cercanas a la plataforma continental en busca de alimento (Markaida \& Sosa 2003), siendo esta zona poco frecuentada por este depredador. Los demás componentes alimentarios encontrados también permiten reconocer la zona oceánica como su área de alimentación, debido a que la mayoría de sus presas se distribuyen allí (i.e. S. sagax, A. thazard, S. oualaniensis, A. affinis y A. lesueurii).

Con relación a la variación mensual del alimento, durante los tres primeros meses (julio a septiembre), la presencia de estas presas ratifica la tendencia del depredador a alimentarse tanto en aguas oceánicas como costeras debido a las características de hábitat de sus presas. Septiembre fue mes de transición debido a que se observó una leve tendencia del depredador a capturar presas de hábitos costeros como Lagocephalus lagocephalus y Ophichthus sp. Durante los meses de octubre a diciembre se evidenció el incremento de presas de zonas oceánicas cercanas a la plataforma 
continental, lo cual se reveló en el mayor consumo de $B$. panamense y cefalópodos como D. gigas y $S$. oualaniensis. Así mismo, aumentó el consumo de presas bentónicas como $M$. gayi que habita los márgenes de la plataforma continental. El cazar presas diferentes en estos meses puede deberse a la ausencia de su principal recurso alimentario L. argenteus.

De acuerdo a la variación del espectro trófico entre hembras y machos de $A$. superciliosus, hay una clara diferencia en cuanto al número de presas, encontrando en las hembras 22 componentes con una preferencia por cuatro de hábitos costeros L. argenteus, D. gigas, M. gayi y B. panamense y las demás especies secundarias y circunstanciales se caracterizaron por ser de hábitos oceánicos lo cual permite inferir que las hembras se alimentan tanto en la zona oceánica como en la plataforma continental; mientras que los machos con 14 componentes alimentarios presentaron sólo dos presas principales $L$. argenteus y $M$. gayi, la mayoría de sus presas secundarias y circunstanciales se distribuyen cerca a la costa, presentando una mayor afinidad a alimentarse en aguas costeras.

Para individuos maduros de ambos sexos se presentó una mayor variedad de presas consumidas (20 en las hembras y 13 en los machos) en comparación con los individuos inmaduros, que presentaron una dieta con menos componentes alimentarios (tres en las hembras y seis en los machos) y aunque dominaron las mismas presas principales, su proporción fue menor. La presencia de presas comunes con diferentes porcentajes de IIR indica que no existe una segregación por estados de madurez, a pesar de la diferencia en la variedad de presas; sin embargo, la capacidad de captura por parte del depredador podría explicar esta diferencia. Lowe et al. (1996) mencionan que los tiburones de mayor tamaño se pueden alimentar de presas grandes, ya que tienen acceso a diferentes hábitats; de la misma forma dependiendo de los diversos estadios de desarrollo ocuparán áreas distintas ya que se segregan por tallas y sexos. Esto explica el por qué los individuos maduros muestran una dieta más variada, constituida por especies oceánicas y costeras ya que tienen acceso a diferentes áreas de alimentación; a diferencia de los inmaduros que presentan en su dieta menos número de presas que son abundantes en la costa. Con relación a la alimentación de las hembras grávidas, se observó mayor preferencia por los peces, posiblemente debido a que en este estado las hembras aumentan en peso corporal lo cual les resta capacidad para cazar presas rápidas como los calamares, prefiriendo capturar presas menos ágiles y que formen cardúmenes. El hecho de que las hembras en estado de gestación se alimenten contradice lo enunciado por varios autores como Cabrera (2000) quien determinó que en otras especies como Carcharhinus falciformes y Nasolamia velox, las hembras grávidas no se alimentan, ya que ahorran con anterioridad suficiente reserva energética para esta fase y prefieren evitar los riesgos y el gasto energético que conlleva la caza.

La baja diversidad en la dieta de A. superciliosus dominada por cuatro presas principales, índica que este tiburón para el área de Manta (Ecuador) se clasifica como un depredador especialista, cuyas presas presentan amplias áreas de distribución y movilidad, además de tener acceso a grandes profundidades gracias a la red vascular maravillosa en la región craneal que le permite mantener altas temperaturas en su cuerpo cuando están en busca de alimento (Weng \& Block 2004).

En cuanto a los traslapamientos, los valores altos entre machos y hembras, y entre machos (maduros e inmaduros) se deben a la mayor abundancia de $L$. argenteus y $M$. gayi, mostrando que no existe segregación entre ellos. Por su parte, los valores bajos en las hembras (maduras e inmaduras) permiten corroborar que existe una segregación entre hembras dependiendo del estado de madurez, presentando sólo dos presas en común L. argenteus y M. gayi, la primera representada en hembras inmaduras que pasan la mayor parte del tiempo en la zona costera, pero migran frecuentemente hacía la zona oceánica corroborado por la presencia de $S$. sagax.

Preti \& Smith (2001) determinaron los hábitos alimentarios de $A$. vulpinus en el área de California, encontrando que esta especie tiene una dieta compuesta en su mayoría por peces como Engraulis mordax, Merluccius productus y S. sagax, las cuales son presas de hábitat costero y bentónico. Es evidente la similitud de la dieta de $A$. vulpinus con $A$. superciliosus ya que capturan peces de hábitat similares; sin embargo, la presencia de un mayor número de presas oceánicas en la dieta de $A$. superciliosus permite reconocer este ambiente como el de mayor preferencia al momento de alimentarse. 


\section{Conclusiones}

El análisis del espectro trófico de A. superciliosus mostró variaciones mensuales en su composición y abundancia, reflejando que entre julio y septiembre este tiburón tiende a alimentarse tanto en la zona oceánica como en la costera, mientras que de octubre a diciembre lo hace únicamente en la segunda; a pesar de lo anterior se detectó a $L$. argenteus, $M$. gayi, D. gigas y $B$. panamense como presas preferenciales.

A través del estudio alimentario no se observó segregación por sexo entre machos y hembras de $A$. superciliosus; sin embargo, se registró segregación entre hembras maduras e inmaduras y se determinó que las grávidas sí se alimentan y lo hacen principalmente de peces oceánicos y costeros.

Por sexo y estado de madurez, se corroboró que en la zona de estudio A. superciliosus se puede clasificar como un depredador especialista, debido al consumo de pocas presas.

\section{Agradecimientos}

A la Comisión Interamericana del Atún Tropical (CIAT) por facilitar sus instalaciones para el almacenamiento de las muestras, al Centro Interdisciplinario de Ciencias del Mar (CICIMAR) por el suministro de los materiales. Al biólogo Jimmy Martínez por su cooperación durante la fase de campo y a las Universidades Eloy Alfaro de Manabí (Ecuador) y Jorge Tadeo Lozano, Sede Santa Marta (Colombia), por permitir el procesamiento del material en sus laboratorios y la preparación de este manuscrito.

\section{Literatura citada}

Alcívar V \& $\quad$ F López. 2001. Pesca artesanal y comercialización de los tiburones zorros Alopias superciliosus (Lowe, 1839) y Alopias vulpinus (Nakamura, 1935) en el Puerto de Manta, Provincia de Manabí. Tesis de Biología Pesquera. Facultad de Ciencias del Mar. Universidad Laica "Eloy Alfaro” de Manabí. Ecuador, 56 pp.

Cabrera A. 2000. Determinación de hábitos alimenticios durante las estaciones primavera y verano de Carcharhinus falciformis, Sphyrna lewini y Nasolamia velox (Carcharhiniformes: Carcharhinidae) a partir del análisis de su contenido estomacal en el golfo de
Tehuantepec, México. Tesis de Licenciatura. Universidad Nacional Autónoma de México. México, 63 pp.

Castillo R, V Blaskovic, F Fernández \& A Alamo. 1996. Características biológicas de la merluza y otras especies demersales en otoño de 1995. Informe Instituto del Mar del Perú 117: 99-107.

Cervigón F \& A Álcala. 1999. Los peces marinos de Venezuela. Tiburones y Rayas. Vol V, 230 pp. Fondo Editorial del Estado de Nueva Esparta. Caracas.

Chen L. 1997. Reproductive biology of the bigeye thresher shark, Alopias superciliosus (Lowe 1839) (Chondrichthyes: Alopiidae), in the northwestern Pacific. Ichthyological Research 44(3): 227-235.

Chirichigno N. 1998. Clave para identificar los peces marinos del Perú. 480 pp. Instituto del Mar del Perú Callao, Perú.

Clarke M. 1962. The identification of cephalopod beaks and their relationship between beak size and total body weight. Bulletin British Museum of Natural History 8 (10): 422-480.

Clarke M. 1986. A handbook for the identification of cephalopod beaks. 273 pp. Clarenron Press, Oxford.

Clothier C. 1950. A key to some Southern California fishes based on vertebral characters. Department of Natural Resources. Division of Fish and Game. Fish Bulletin 79, 83 pp.

Clothier C \& J Baxter. 1969. Vertebral characters of some Californian fishes with notes on other Eastern Pacific species. Department of Fish and Game. Marine Resources Operations. State of California, 32 pp.

Compagno L. 1984. Sharks of the world. An annotated and illustrated catalogue of shark species known to date. Part 1. Hexanchiformes to Lamniformes. FAO Fisheries Synopsis 4: 1-655.

Compagno L. 2001. Species catalogue the shark of the world. An annotated and illustrated catalogue of shark species known to date. FAO Species Catalogue for Fishery Purposes 1(2): 1-269.

Espinoza P. 2001. Composición de la dieta de la merluza Merluccius gayi peruanus a comienzos del verano de 1999. Crucero BIC José Olaya Balandra 9901. Instituto del Mar del Perú. En prensa.

Fischer W, F Krupp, W Schneider, C Sommer, K Carpenter \& VH Niem. 1995. Guía FAO para la identificación de especies para los fines de pesca. Pacífico 
Centro Oriental. Vol II. Vertebrados parte 1 y 2: 6471813.

Galván F, H Nienhuis \& P Klimley. 1989. Seasonal abundance and feeding habits of sharks of the lower Gulf of California. Mexico. California Fish and Game 75: 7484.

García I. 2001. Patrones morfológicos del otolito Sagitta de algunos peces óseos del mar peruano. Instituto del Mar del Perú 20 (1-2): 4-83.

Garth J \& W Stephenson. 1966. Brachyura of the Pacific coast of America. Brachyrhyncha: Portunidae. Allan Hancock Monographies. Marine Biology 1:1-154.

Hyslop E. 1980. Stomach contents analysis: a review of methods and their application. Journal Fisheries Biology 17: 411-429.

Instituto Nacional de Pesca de Ecuador. 1998. Desembarques pesqueros en el Puerto de Manta. Informe Técnico No 1, 40 pp.

Krebs C. 1989. Ecological methodology, 550 pp. Harper \& Row, New York.

Labropoulou M \& A Eleftheriou. 1997. The foraging ecology of two pairs of congeneric demersal fish species: importance of morphological characteristics in prey selection. Journal of Fish Biology 50: 324-340.

Langton R. 1982. Diet overloap between the Atlantic cod Gadus morhua, silver hake Merluccius bilinearis and fifteen other Northwest Atlantic finfish. Fish Bulletin 80: 745-759.

Liu K, P Chiang \& C Chen. 1999. Age and growth estimates of the bigeye thresher shark, Alopias superciliosus, in northeastern waters. Fish Bulletin 96: 482-491.

López C, L Ochoa \& F Gonzáles. 1999. Estadísticas de los desembarques pesqueros en Ecuador 1985-1997. Boletín Científico Técnico del Instituto Nacional de Pesca 27 (4): 20-27.

Lowe G, G Wetherbe, G Crow, A Wetherbe G Crow \& A Tester. 1996. Ontogenetic dietary shifts and feeding behavior of the tiger shark Galeocerdo cuvier, in Hawaiian waters. Environmental Biology Fish 47: 203211.

Markaida U \& O Sosa. 2003. Food and feeding habits of jumbo squid Dosidicus gigas (Cephalopoda: Ommastrephidae) from the Gulf of California, Mexico. Journal of Marine Biology 83: 507-522.

Martínez J. 1998. Caso de estudio sobre el manejo de las pesquerías de tiburones en el Ecuador. Guayaquil. Ecuador. Informe Técnico para la FAO: 12-25.

Moreno J \& J Morón. 1992. Reproductive biology of the bigeye thresher shark, Alopias superciliosus (Lowe 1839). Journal of Marine \& Freshwater Research 43: 7786.

Pinkas L, M Oliphant \& L Iverson. 1971. Food habits of albacore, bluefin tuna, and bonito in California waters. Department Fish and Game. Fish Bulletin 152, 105 pp.

Preti A \& S Smith. 2001. Feeding habits of the common thresher shark (Alopias vulpinus) sampled from the California-based drift net fishery, 1998-1999. California Department of Fish and Game. CaLCOFI Report 42: 145152.

Smith P \& M Zaret. 1982. Bias in estimating niche overlap. Ecology 63 (5): 1248-1253.

Weng K \& B Block. 2004. Diel vertical migration of the bigeye thresher shark (Alopias superciliosus), a species possessing orbital retia mirabilia. Fishery Bulletin 102 (1): 221-229.

Wolff C. 1982. A beak key for eight eastern tropical Pacific cephalopods species, with relationship between their beak dimension and size. Fish Bulletin 80 (2): 357-370.

Wolff C. 1984. Identification and estimation of size from beaks of eighteen species of cephalopods from the Pacific Ocean.. NOAA Technical Report. NMFS. 17, 41 pp.

Wooton, R. 1990. Ecology of teleost fishes, 404 pp. Chapman \& Hall, New York. 